\section{Arznei wird oft nicht korrekt eingenommen}

Für eine französische Studie wurden 128 Patienten und deren Ärzte zur Wichtigkeit der verordneten 498 Präparate und zur Einnahmetreue befragt. 18,9\% der verordneten und von den Ärzten als wichtig eingestuften Präparate wurden nicht korrekt eingenommen. Die Wissenschaftler empfehlen, regelmäßig für langfristig verordnete Medikamente zu prüfen, ob die Patienten es einnehmen. Bei schlechter Compliance sei das RisikoNutzen-Verhältnis abzuwägen und ggf. auch einmal auf eine weniger bedeutende, aber neben- und wechselwirkungsträchtige Verordnung zu verzichten.

Sidorkiewicz S. et al. Ann Fam Med. 2016; 14: 415-421

\section{Bauchgefühl erkennt den Krebs}

Für eine niederländische Studie wurden Allgemeinärzte aufgefordert, einen Fragebogen auszufüllen, wenn sie bei einem ihrer Patienten, unabhängig von klinischen Zeichen, den Eindruck hatten, es könne ein Krebs vorliegen. Jeweils drei Monate später wurde dann die tatsächliche Diagnose evaluiert. 59 Ärzte berichteten bei $366 \mathrm{~Pa}$ tienten über einen solchen intuitiven Krebsverdacht. Drei Monate später war der Verdacht bei $35 \%$ der Patienten bestätigt. Hausärzte über 50 hatten sogar in $43 \%$ der Fälle das richtige Gespür gehabt.

Donker GéA et al. BMJ Open 2016;6:e012511 doi:10.1136/bmjopen-2016-012511

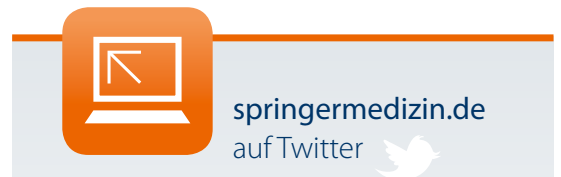

Auch im Herbst heißt es wieder:

Lauschen Sie unserem Zwitschern und werden Sie zum Follower!

twitter.com/springermedizin

Bannwarth-Syndrom

\title{
Heilungschancen bei der Neuroborreliose
}

\begin{abstract}
Patienten mit Neuroborreliose haben heutzutage meist weniger Komplikationen als vor Jahrzehnten - offenbar wird die Diagnose inzwischen deutlich früher gestellt.
\end{abstract}

Slowenische Forscher haben untersucht, wie gut die Therapie einer Neuroborreliose anschlägt. Innerhalb von acht Jahren untersuchten sie 77 Patienten mit Bannwarth-Syndrom. Alle unterzogen sich einer zwei- bis dreiwöchigen Therapie mit Ceftriaxon oder Doxycyclin. 14 Tage nach Beginn der Antibiose waren die Symptome bei $88 \%$ der Patienten fast oder komplett verschwunden. 26\% der Betroffenen erhielten aufgrund einer anhaltenden Liquor-Pleozytose und/oder Beschwerden eine erneute Antibiose, 12\% hatten langfristig Beschwerden. Im Schnitt wurde

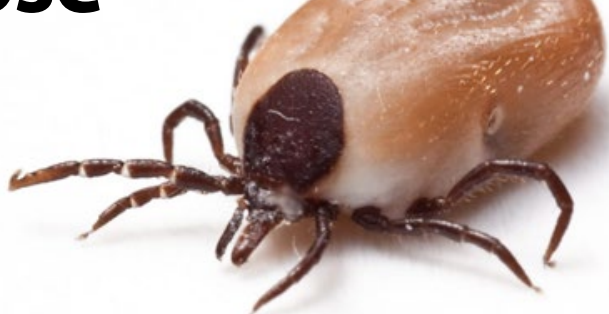

eine Neuroborreliose 30 Tage nach Beginn der neurologischen Symptome diagnostiziert. Dies sei immer noch zu spät, aber doch wesentlich früher als im vergangenen Jahrhundert, so die Ärzte. Auch sei eine periphere Fazialisparese nur noch halb so oft beobachtet worden wie in früheren Untersuchungen, ausgeprägte Lähmungen traten zu $80 \%$ seltener auf. Dies spreche dafür, dass schwerwiegende Komplikationen dank der früheren Diagnose inzwischen relativ selten sind.

(mut)

Ogrinc K. et al. Clin Infect Dis. 2016;63(3):346-53.

Typ-2-Diabetes

\section{Hoher $\mathrm{HbA}_{1 c}$-Wert freut Bakterien und Pilze}

Typ-2-Diabetiker leiden häufiger als Nichtdiabetiker an Infektionskrankheiten. In einer britischen KohortenStudie machten diesen Unterschied v.a. Erkrankungen aus, die durch Bakterien oder Pilze verursacht werden.

Forscher um Julia Hine von der University of Surrey in Guildford in Großbritannien und ihre Kollegen haben die Infektionsraten des Jahres 2014 von 34.278 Patienten mit und 613.052 Patienten ohne Typ-2-Diabetes verglichen. Dabei wurden Infektionen der oberen Atemwege, Bronchitiden, grippeähnliche Erkrankungen, Pneumonien, infektiöse Darmerkrankungen, Herpes-simplex-Virus (HSV), Haut- und Weichteilinfektionen, Harnwegs-, Genitalund perineale Infektionen berücksichtigt.
Die Diabetiker hatten im Vergleich zu den Nichtdiabetikern v. a. vermehrt bakterielle und Pilzinfektionen. Außer der HSVInfektion traten alle untersuchten Infektionskrankheiten bei Diabetikern häufiger auf als bei Nicht-Diabetikern (altersstandardisierte Gesamtinfektionsrate: 502,0 vs. 267,3 pro 1000 Personenjahre).

\section{Signifikant häufiger Infektionen}

Patienten mit einem $\mathrm{HbA}_{1 \mathrm{c}}$-Wert > 8,5\% hatten im Vergleich zu solchen mit einem $\mathrm{HbA}_{1 \mathrm{c}}$-Wert $<7 \%$ signifikant häufiger Bronchitiden, Pneumonien, Haut- und Weichteilinfektionen, Harnwegsinfekte sowie Genital- und perineale Infektionen.(St)

Hine J L et al. Diabet Med 2016, online 22. August, DOl: 10.1111/dme.13205 